\title{
Testing solar panels for small-size satellites: the UPMSAT-2 mission
}

\author{
E Roibás-Millán $\oplus^{1}$, A Alonso-Moragón, A G Jiménez-Mateos \\ and S Pindado
}

Instituto Universitario de Microgravedad Ignacio Da Riva (IDR/UPM), Universidad Politécnica de Madrid, 28040, Madrid, Spain

E-mail: elena.roibas@upm.es

\begin{abstract}
At present, the development of small-size satellites by universities, companies and research institutions has become usual practice, and is spreading rapidly. In this kind of project cost plays a significant role. One of the main areas are the assembly, integration and test (AIT) plans, which carry an associated cost for simulating environmental conditions. For instance, in the power subsystems test and, in particular, in the testing of solar panels, the irradiance and temperature conditions might be optimum so the performance of the system can be shown next to real operational conditions. To reproduce the environmental conditions in terms of irradiance, solar simulators are usually used, which carries an associated increase in cost for testing the equipment. The aim of this paper is to present an alternative and inexpensive way to perform AIT plans on spacecraft power subsystems, from a testing campaign performed using outdoor clean-sky conditions and an isolation system to protect the panels. A post-process of the measured data is therefore needed, taking into account the conditions in which the test has been accomplished. The $I-V$ characteristics obtained are compared with a theoretical 1-diode/2-resistor equivalent electric circuit, achieving enough precision based solely on the manufacturer's data.
\end{abstract}

Keywords: solar cell, solar panels testing, space power system, micro-satellites

(Some figures may appear in colour only in the online journal)

\section{Introduction}

Since the beginning of the astronautic era, photovoltaic devices have been considered for the generation of electrical power on-board spacecraft because of their high power output per unit mass. Their simplicity, relatively modest cost, and high reliability have caused this system to be chosen to supply sustained electrical power for almost all unmanned spacecraft.

Solar cells for space applications have to be highly efficient and capable of standing thousands of thermal cycles in orbit where the temperature, according to the mission profile, may vary from $-150{ }^{\circ} \mathrm{C}$ to more than $120^{\circ} \mathrm{C}$. Cell materials have to show a limited degradation over time due to cosmic radiation and be capable of resisting mechanical solicitations, mainly linear accelerations and vibrations, during launch and orbital manoeuvres. In the past, silicon cells were the most common photovoltaic technology in space; the reachable bulk efficiency was not higher than $14 \%$. The advent of GaAs solar cells in the last decade of the 20th century improved efficiency up to $19 \%$, whereas triple junction solar cells reach about $30 \%$ efficiency levels [1].

In the few last years an increasing interest in the development of small size satellites has arisen; these small spacecraft being usually developed by research institutes and universities where the lifecycle cost must be minimized [2]. Even slight improvements in development, production and qualification processes may result in significant savings for small satellites' programs, and in particular for programs developed at the universities, where cost plays a significant role.

Low-cost methods for solar panel assembling, and the development of a flexible and simple array to be mounted on generic small satellite platforms, are still open research topics. Usually, for small size satellites, solar panels are manufactured using commercial solar cells or modules, connecting 
them in series or in parallel depending on the system requirements. After assembly operations, the performance of the solar panels must be evaluated by functional testing to identify any malfunction that may occur, as most of the failure issues regarding spacecraft power subsystems are related to the solar arrays [3, 4]. These tests should include solutions with high flexibility and reduced cost, avoiding the need for dedicated and expensive technologies and facilities.

The most critical element in the testing of solar cells is the light source, also becoming the major source of error $[5,6]$. Under the right atmosphere conditions (usually, low-air-mass and clean sky), the spectrum of natural sunlight has been revealed as an excellent match to the standard spectra [7]. Some works have performed several comparisons between different artificial light sources (solar simulators) and natural sunlight [8]. It seems that, at present, the spectrum of natural sunlight is a better match to the AM1.5G standard spectra [9] than a lowcost solar simulator, and similar to an expensive one.

Besides, it can be pointed out that in a quite recent intercomparison between reference laboratories, the results of photovoltaic performance analysis using sunlight were as accurate as the ones obtained using a precise solar simulator [10]. With regard to the cost disadvantages of using solar simulators, Carrillo et al [11] state that they can easily exceed $€ 150000$, whereas sunlight testing of photovoltaic devices can be carried out with inexpensive equipment.

If the comparison between photovoltaic performance analysis using sun simulators or sunlight is based on the procedure, both methods seem to be equally demanding in terms of lab resources $[12,13]$. Apparently, sunlight testing could be considered to be simpler but this is a misconception, as the outdoor testing requires calibrated reference solar cells, control over thermal effects, and one to take into account the effects of spectral reflectance $[12,14,15]$.

Sun simulators are normally used in the space industry. After a review of the available literature, the first example found in relation to spacecraft solar array testing is the SERT II mission, which was carried out by using lamps [16]. It seems that within the space industry, the testing of solar cells is carried out after qualification testing [17], environmental exposure degradation testing [18], or by studying solar array performance under special environmental conditions such as close-to-the-Sun or far-from-the-Sun missions [19, 20]. Obviously, missions in which new photovoltaic technologies are used require thorough testing campaigns, carried out by using sunlight simulators [21].

The present work is focused on performance testing and evaluation of UPMSAT-2 micro-satellite solar panels after assembly operation. This performance evaluation is carried out by means of an inexpensive $I-V$ curve determination procedure using outdoor clean sky measurements and without using calibrated reference cells. It should be highlighted that the outdoor procedure to test UPMSAT-2 solar arrays is unusual within the space industry. Outdoor or sunlight testing was developed by the photovoltaic solar energy industry to meet real operating conditions $[22,23]$ and, to the authors' knowledge, spacecraft solar arrays are not tested using outdoor sunlight.
Table 1. UPMSAT-2 mission main parameters.

\begin{tabular}{ll}
\hline Parameter & Description \\
\hline Mission life & 2 years \\
Orbit type & Sun-synchronous/Noon \\
Orbital altitude & $700 \mathrm{~km}$ \\
Orbit period & $\approx 98 \mathrm{~min}($ eclipse time $36 \mathrm{~min})$ \\
Satellite mass & $<50 \mathrm{~kg}$ \\
Satellite geometrical envelope & $0.5 \times 0.5 \times 0.6 \mathrm{~m}^{3}$ \\
\hline
\end{tabular}

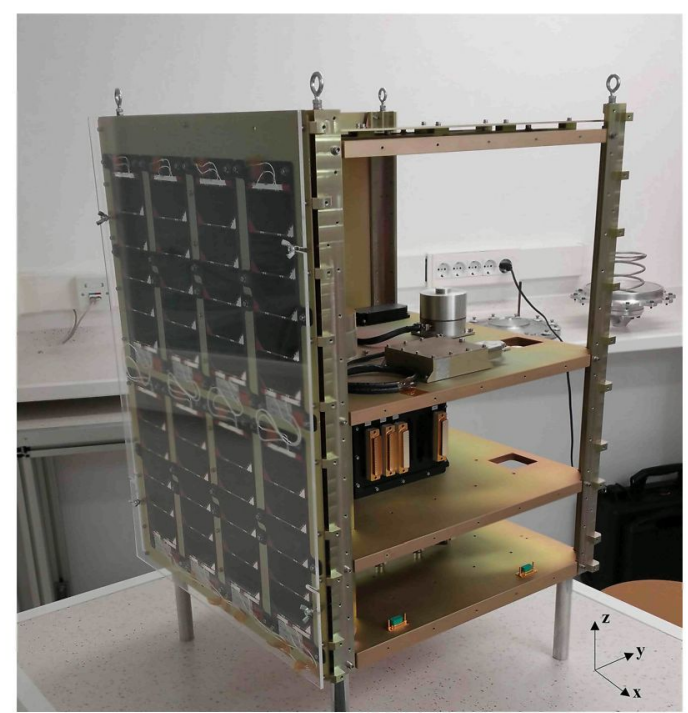

Figure 1. Picture of the UPMSAT-2 flight model during AIT/AIV operations.

One important factor to take into account is that the characteristic $I-V$ curves and the power produced by a photovoltaic device is highly dependent on the environmental conditions, namely the temperature and the solar irradiance. Although several numerical models are available to forecast the operation of solar panels, a problem arises on the definition of the parameters for many of these models. The data provided by a manufacturer's datasheet usually contains only information at standard conditions or for the nominal operating cell temperature. In the case of space solar panels, this fact is of particular significance, since the space environment (in terms of temperature and solar irradiance spectrum) is usually difficult to reproduce in terrestrial conditions.

To overcome this problem, the outdoor testing results in this work were post-processed taking into account the environmental conditions, in order to compare the resulting $I-V$ curves with the ones based on the manufacturers' data.

This paper is organized as follows, section 2 is focused on the UPMSAT-2 mission description and, in particular, on the solar panels used as the power source. In section 3, particular considerations taken into account to perform the tests are presented, whereas the testing set-up is described in section 4 . Finally, section 5 is focused on the comparison between the measured $I-V$ curves and the ones based on the equivalent circuit models, to conclude with a discussion and conclusions of the work in section 6 . 
Table 2. Payloads on-board UPMSAT-2.

\begin{tabular}{lll}
\hline Component & Company & Power requirements \\
\hline Micro thermal switch & IberEspacio & $5 \mathrm{~W}(10 \mathrm{~min}$ at start $) / 40 \mathrm{~W}(\max )$ \\
Magnetometer & Bartington & $0.53 \mathrm{~W}(\max )$ \\
Reaction Wheel & SSBV & $16 \mathrm{~W}(\max )$ \\
CTM (Thermal control experiment) & IDR/UPM & $\mathrm{W}$ \\
SS6 (Solar sensor system) & IDR/UPM & $1 \mathrm{~W}$ \\
MRAD (Monitoring the Radiation Effects) & STRAST/UPM & $0 \mathrm{~W}$ \\
\hline
\end{tabular}

\section{The UPMSAT-2 mission}

The UPMSAT-2 is a $50 \mathrm{~kg}$-class microsatellite developed for a 2-year LEO mission. This satellite will be orbiting in a sunsynchronous orbit at around $700 \mathrm{~km}$ altitude. This mission is preceded by a former one, the UPMSat-1, launched from French Guiana on 7 July 1995, and is fully developed by the same team $[24,25]$. The UPMSat-1 travelled into space as secondary payload on flight V75 launcher Ariane IV-40, 213 days being the in-orbit operational life.

The UPMSAT-2 is a scientific and technology demonstration satellite orbiting the Earth, where educational character is a key goal [26]. The main parameters of the satellite are summarized in table 1, and a picture of the satellite is shown in figure 1.

The UPMSAT-2 will be orbiting in a sun-synchronous noon orbit with the $Z$-axis perpendicular to the orbit plane. The power consumption is limited by the available power obtained by the sun radiation. The list of on-board payloads are summarized in table 2, where their power consumption requirements are included.

The on board data handling (OBDH) subsystem (represented by the on-board computer and their major components), and the on-board buses and communication protocols are designed by Tecnobit and STRAST/UPM ${ }^{1}$. The attitude control and determination subsystem (ADCS) is based on Earth's magnetic field interaction, and it is composed by magnetic torquers (ZARM Technik AG) and magnetometers (SSBV Space \& Ground Systems). The UPMSAT-2 power system is composed of five fixed solar panels, a Li-Ion battery and the power distribution subsystem (PDS). Four panels are disposed at the lateral sides of the cuboid, whereas the fifth is located at the top side. These panels are built with Selex Galileo SPVS-5S solar modules. The battery was designed and manufactured by SAFT, being based on the Li-Ion technology represented by VES-16 cells.

\subsection{The UPMSAT-2 solar panels system}

As aforementioned, the UPMSAT-2 solar panels are built with SPVS-5S solar modules, composed of triple junction solar cells (AZUR SPACE 3G28C, efficiency class 28\%) mounted on an $\mathrm{Al}$ substrate, instead of a traditional one integrated on to a composite CFRP + Al substrate. The selected substrate is made of black anodized aluminium in order to guarantee complete insulation towards the electrical network. Each module

\footnotetext{
${ }^{1}$ http://web.dit.upm.es.
}

is composed of five solar cells and it is autonomous in terms of the supporting structure and connection terminals, as a blocking diode can be directly mounted at the end of the last module for each string.

The lateral solar panels $(X+, X-, Y+$ and $Y-)$ are formed by four parallel-connected groups of two series-connected SPVS-5S modules, while the solar panel at the top side $(Z+)$ is formed by two parallel-connected groups of two series-connected SPVS-5S modules (see figure 2). The panel $Z+$ will not receive any irradiation from the sun in the normal operational mode. Nevertheless, it represents an extra energy source at the initial stage of the mission, just after the satellite deployment at the orbit, and also when the satellite attitude at the normal operation mode is altered by the reaction wheel testing.

The SPVS-5S modules are directly fixed on the lateral support panels of the satellite, which are fabricated in aluminium EN AW 7075 and coated with Alodine ${ }^{\circledR} 1200$ to minimize corrosion. Areas without surface treatment are also covered with 1 Mil Kapton ${ }^{\circledR}$ tape. Figure 2 shows the final appearance of both configurations. A blocking diode is included in each of the solar cells' series, with a forward voltage of $0.8 \mathrm{~V}$.

In table 3, the manufacturer's datasheet for solar cells and modules that compose the UPMSAT-2 solar panels are presented.

\section{UPMSAT-2 Solar Panels' performance measurements}

The performance of a solar cell or module is characterized by the output power at a maximum power point, $P_{\mathrm{mp}}$, which is usually determined by varying the forward bias voltage across the device under test while illuminated [27]. The characteristic $I-V$ curve is then generated by measuring the current and voltage. Usually, the determination of $P_{\mathrm{mp}}$ is complex because it depends on the irradiance incident upon the device, its spectrum and the cells temperature. Accurate determination of solar panel performances requires knowledge of the potential measurement problems, the way these problems are influenced by the specific specimen to be tested and how they affect the solar panel performances.

In figure 3 the block diagram for a typical $I-V$ curve determination is shown. The photovoltaic device under test is illuminated, the load is varied, and the operating point of the device under test changes, which allows one to measure the current and voltage along the $I-V$ curve. A Kelvin connection to the device is used, allowing the voltage across the device to be measured by avoiding voltage drops along the wiring in the current measurement loop. 

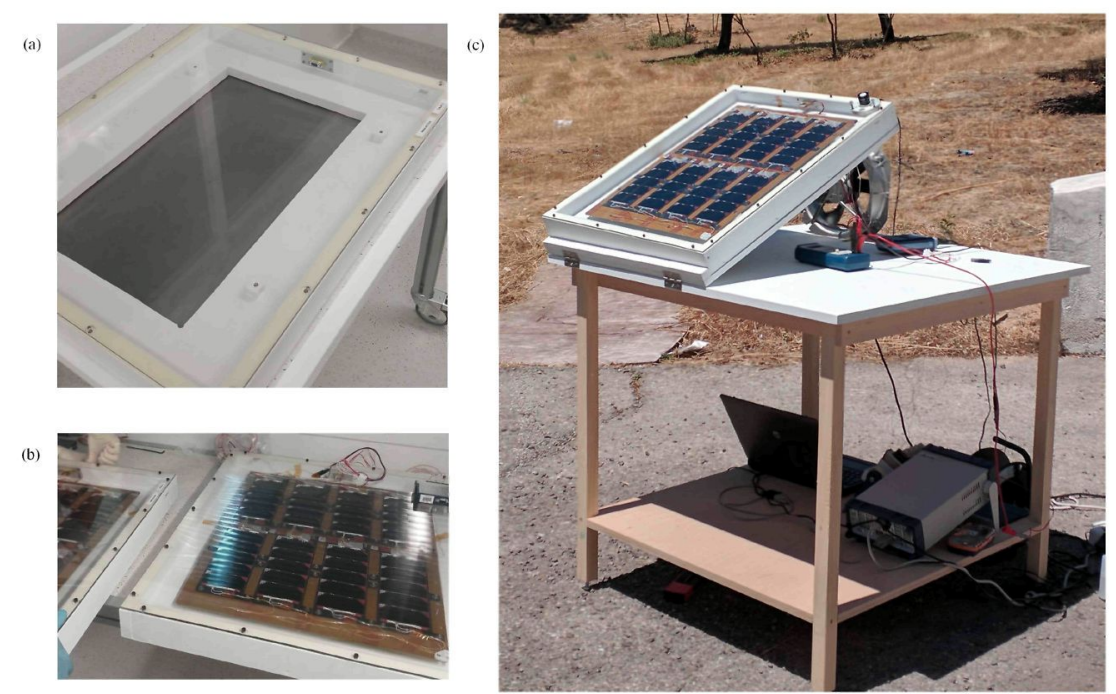

Figure 4. (a) Support device used for solar panels testing; (b) a detail of the solar panels integration inside the support box; and (c) test instrumentation for outdoor measurements.

It should be emphasized that the standardized spectral irradiances cannot be reproduced exactly in the laboratory (with solar simulators or natural sunlight). Thus, $I-V$ curves calculated from measurements shall be normalized to AM0 irradiance values. Additionally, identification of the spectral irradiance of terrestrial measurements is a difficult task, because of the effects of the atmosphere on sunlight, which are a function of the path length through the atmosphere [35].

The American Society for Testing and Materials (ASTM) defines two standard terrestrial spectral distributions, the direct-normal and global AM1.5 spectrum [9]. The receiving surface is defined in the standards as an inclined plane at $37^{\circ}$ tilted toward the equator, facing the sun (i.e. the surface normal points to the sun, at an elevation of $41.81^{\circ}$ above the horizon). The direct-normal standard, AM1.5D, corresponds to the incident radiation that perpendicularly reaches a sun-facing surface directly from the Sun. However, the spectral content of solar radiation at the Earth's surface has a diffuse component in addition to the direct, due to reflection and scattering in the atmosphere. The standard AM1.5G represents the global spectrum, which is designed for flat plate modules, and is made from both diffuse (scattered) and direct sunlight, which has become the testing and comparison standard for terrestrial photovoltaic applications.

\section{Solar panels' testing}

As mentioned in the previous section, solar panels experimental $I-V$ curves are determined through measurements of the output voltage and output current variations, as sketched in figure 3. The set of measurements is performed from the solar panel open-circuit point, where the output current will be at its minimum and the output voltage reaches its maximum value, i.e. $V_{\mathrm{oc}}$. From this testing condition, the output current is increased until its maximum value is reached, i.e. $I_{\mathrm{sc}}$, when the solar panel is short-circuited and the output voltage is at its minimum value, i.e. zero.

\subsection{Testing set-up}

The UPMSAT-2 solar panels' measurement system follows the aforementioned description. The variable load used for the test can be as simple as a variable resistor. However, a computerprogrammable variable load is used, a BK Precision ${ }^{\circledR} 8500$ model, allowing both to obtain current values in any range needed, and the corresponding output voltage measurements. At the beginning of each test, $I_{\mathrm{sc}}$ and $V_{\mathrm{oc}}$ are measured with a digital multimeter PROMAX ${ }^{\circledR}$ PD-181, previously calibrated.

The solar panels are encapsulated in a support device made up of a white painted wooden box and a methacrylate cover to allow sunlight to reach the solar cells. For temperature monitoring, a set of thermocouples are placed along the solar cells modules and an acquisition system in LabView ${ }^{\circledR}$ is used in order to monitor temperatures during each test realization. A temperature-controlled plate is located in the back side of the support to dissipate the heat inside the box and it is continuously refrigerated by an air flow to keep the temperature as stable as possible during the test campaign. The whole device support is shown in figure 4 .

Any light source used (i.e. natural sunlight or a solar simulator) would have temporal instabilities, spatial non-uniformities, drift of its spectral irradiance and an illumination time. In the present work, outdoor clean sky measurements were selected, which normally involve uniform illumination on the test plane (less that $1 \%$ spatial variation), and stable irradiance for quite large time periods (up to several minutes). The support device was mounted on a table that can be tilted so that the angle relative to the sun's rays can be modified (see figure 4(c)). The solar panel support was oriented so that its surface was normal to the direction of the sun, the test campaign being carried out at a $0^{\circ}$ solar zenith angle. In addition, a luxmeter Delta $\mathrm{OHM}^{\circledR}$ HD2102, located in the solar panel plane, was used to obtain the average incident irradiance under test conditions.

During each test realization, the irradiance levels and solar panels' temperature were monitored to reference all experimental data to the conditions specified in the manufacturer's datasheet. In addition, a temperature variation test 


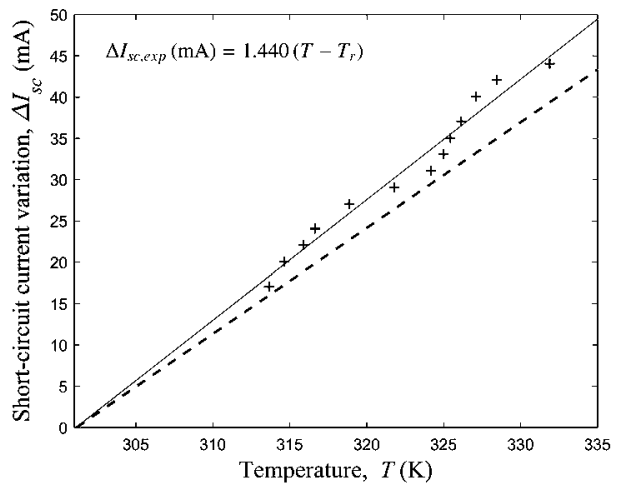

(a)

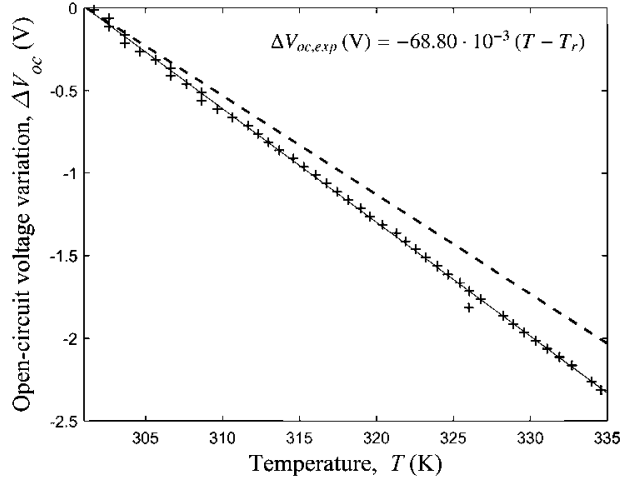

(b)

Figure 5. Variations of (a) the short circuit current and (b) the open circuit voltage with the temperature obtained from the tests, and their comparison with the manufacturer's datasheet. In both graphs: $(--)$ the specified temperature variations given by the manufacturer; $(++)$ the experimental data; and (- the linear fittings of the experimental measures, whose equations are also included in the graphs.

Table 4. Parameter variation with the temperature for a lateral solar panel: experimental determination and manufacturer's data.

\begin{tabular}{lll}
\hline & $\begin{array}{l}\text { Experimental } \\
\text { results }\end{array}$ & $\begin{array}{l}\text { Manufacturer's } \\
\text { data }\end{array}$ \\
\hline$\Delta I_{\mathrm{sc}} / \Delta T \uparrow\left(\mathrm{mA} \mathrm{K}^{-1}\right)$ & 1.440 & 1.280 \\
$\Delta V_{\mathrm{oc}} / \Delta T \uparrow\left(\mathrm{mV} \mathrm{K}^{-1}\right)$ & -68.80 & -60.00 \\
\hline
\end{tabular}

was performed to experimentally determine the characteristic points dependence on the temperature, and compare the results with the data specified at the manufacturer's datasheet.

\section{2. $I_{s c}$ and $V_{o c}$ characteristic points dependence on the temperature}

The mentioned temperature variation was studied on one of the lateral solar panels, the short circuit current, $I_{\mathrm{sc}}$, and the open circuit voltage, $V_{\mathrm{oc}}$, being measured at different temperatures.

The manufacturer's datasheet (see table 3 ) defines the variations of $I_{\mathrm{sc}}$ and $V_{\mathrm{oc}}$ with the temperature for one cell. For a lateral solar panel (with four parallel-connected groups of two series-connected SPVS-5S modules), these variations can be expressed as seen in equations (1) and (2).

$\Delta V_{\mathrm{oc}}(T)=V_{\mathrm{oc}}(T)-V_{\mathrm{oc}}\left(T_{\mathrm{r}}\right)=-60.00 \cdot 10^{-3}\left(T-T_{\mathrm{r}}\right)$

$\Delta I_{\mathrm{sc}}(T)=I_{\mathrm{sc}}(T)-I_{\mathrm{sc}}\left(T_{\mathrm{r}}\right)=1.280 \cdot 10^{-3}\left(T-T_{\mathrm{r}}\right)$

where $T_{\mathrm{r}}=28^{\circ} \mathrm{C}$ is the reference temperature of the data, so that $V_{\mathrm{oc}}\left(T_{\mathrm{r}}\right)=26.67 \mathrm{~V}$ and $I_{\mathrm{sc}}\left(T_{\mathrm{r}}\right)=2.024 \mathrm{~A}$.

As $I_{\mathrm{sc}}$ and $V_{\mathrm{oc}}$ depend on the irradiance reaching the solar panel, and the testing conditions are different from the manufacturer's reference values, the comparison should be performed not in terms of absolute values for $I_{\mathrm{sc}}(T)$ and $V_{\mathrm{oc}}(T)$, but in terms of parameter variation, i.e. $\Delta I_{\mathrm{sc}}(T)$ and $\Delta V_{\mathrm{oc}}(T)$.

The parameter variation from the experimental results, along with the information extracted from the manufacturer's datasheet are shown in figure 5 and table 4 . In the graphs, the linear fitting to the experimental data are included. It should be noted that $\Delta V_{\mathrm{oc}}$ is a negative value as the open circuit voltage decreases as temperature increases.

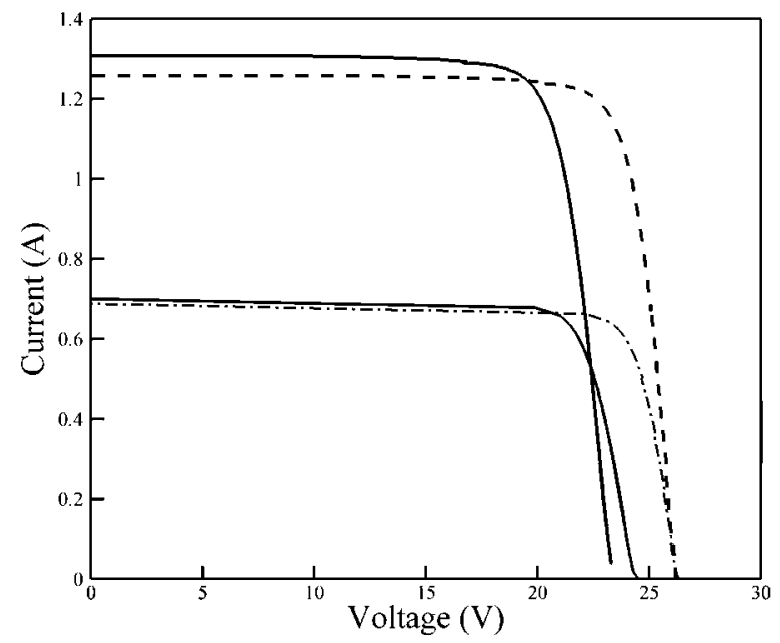

Figure 6. Modifications of the measured $I-V$ curves with the temperature. (-) Lateral solar panel $Y+$ at test temperature; (-) Top panel $Z+$ at test temperature; (- - -) Lateral solar panel at reference temperature; and (-. -) Top panel at reference temperature.

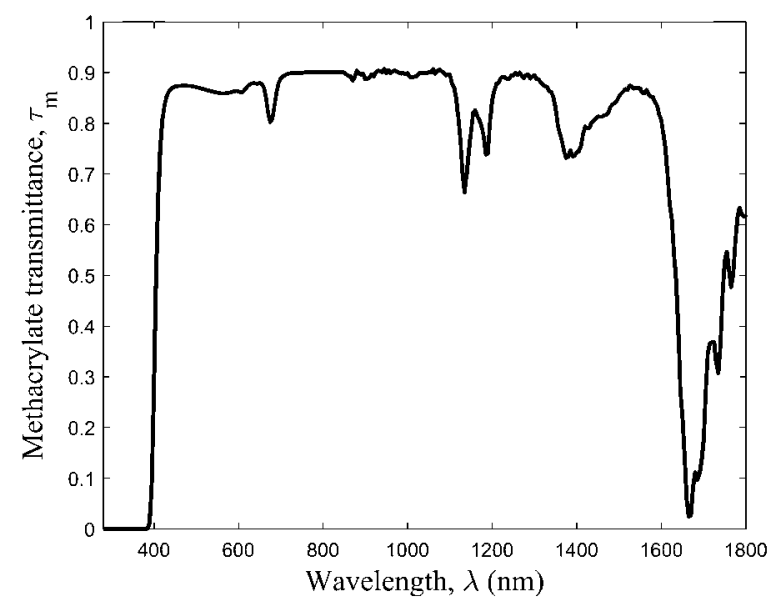

Figure 7. Transmittance of the methacrylate plate protecting the UPMSAT-2 solar panels during tests.

As can be observed in figure 5 , the measured data correlate quite well with the linear variation based on the data specified by the manufacturer in the solar cells' datasheet, the slope 


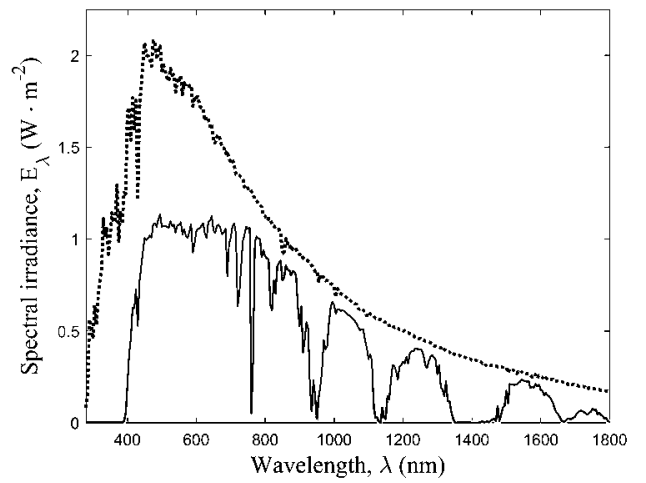

(a)

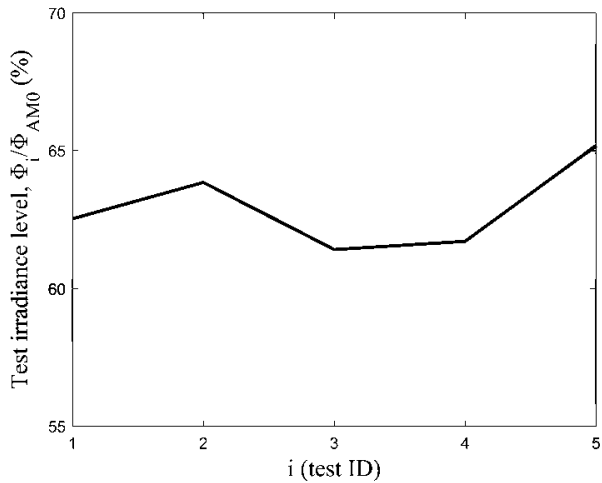

(b)

Figure 8. (a) Spectral irradiance as a function of the wavelength: ( $)$ ) results from the test campaign; ( $\longrightarrow$ ) the mean value of testing measurements; and (---) the AMO standard. (b) Total irradiance levels during tests referenced the AMO spectrum, expressed in percentage. The test IDs from 1 to 5 represent the test conditions for panels $Y+, Y-, X+, X-$ and $Z+$, respectively. The mean value corresponds with a total irradiance of $\Phi_{m}=837.8 \mathrm{Wm}^{-2}$

Table 5. Parameters of 1-diode/2-resistor circuit model of the UPMSAT-2 solar panels. An initial estimation of $a(a=1.1)$ is used.

\begin{tabular}{llllll}
\hline & $a$ & $R_{s}(\Omega)$ & $R_{\mathrm{sh}}(\Omega)$ & $I_{0}(\mathrm{~A})$ & $I_{\mathrm{ph}}(\mathrm{A})$ \\
\hline Lateral panels & 1.1 & 0.044 & $4.246 \cdot 10^{3}$ & $6.024 \cdot 10^{-14}$ & 2.024 \\
Top panel & 1.1 & 0.089 & $8.492 \cdot 10^{3}$ & $3.012 \cdot 10^{-14}$ & 1.012 \\
\hline
\end{tabular}

of the linear expressions being similar to the measured and manufacturer's specified variations. The temperature variations are applied to the measured $I-V$ curves, to normalize the values at test temperature to the manufacturer's reference one. Results for a lateral panel and the top panel are presented in figure 6.

\subsection{Total irradiance under test conditions}

The information provided by the solar cells manufacturer refers to the AM0 irradiance spectrum, whereas the incident solar irradiance over the solar panels in the test environment is considered to be closer to the AM1.5G irradiance spectrum [9]. As aforementioned, the $I-V$ parameters derived from the test should refer to the AM0 standard, for comparison purposes. Therefore, some post-processing of the results is required in the present work.

The total irradiance $E_{S A}$ over the solar panels is expressed as

$$
E_{S A}=\int_{\lambda_{0}}^{\lambda_{1}} E_{\lambda}(\lambda), \mathrm{d} \lambda
$$

where $E_{\lambda}(\lambda)$ is the spectral irradiance, expressed in $\mathrm{W} \mathrm{m}^{-2}$ $\mathrm{nm}^{-1}$, and $\lambda$ is the wavelength. The definite integral of equation (3) must be calculated in the wavelength spectrum where the solar cells used (GaInP/GaAs/Ge) have an efficiency over $5 \%$, i.e. $\left[\lambda_{0}, \lambda_{1}\right]=[300-1750] \mathrm{nm}$.

The spectral irradiance $E_{\lambda}(\lambda)$ reaching the solar panels in the test conditions depends on the atmospheric transmittance and the transmittance of the methacrylate plate protecting the solar panels during the test campaign.
Table 6. Obtained parameters from the theoretical model based on the irradiance values measured by the luxmeter during the test campaign. From top to bottom: values for panels $Y+, Y-, X+, X-$ and $Z+$.

\begin{tabular}{llllll}
\hline Test ID & $\begin{array}{l}\Phi_{i} \\
\left(\mathrm{~W} \mathrm{~m}^{-2}\right)\end{array}$ & $\begin{array}{l}\Phi_{i} / \Phi_{\mathrm{AM} 0} \\
(\%)\end{array}$ & $I_{\mathrm{sc}}(\mathrm{A})$ & $V_{\mathrm{oc}}(\mathrm{V})$ & $\begin{array}{l}P_{\mathrm{mp}} \\
(\mathrm{W})\end{array}$ \\
\hline 1 (Panel $Y+$ ) & 854.09 & 62.52 & 1.27 & 26.67 & 28.96 \\
2 (Panel $Y-)$ & 871.84 & 63.84 & 1.29 & 26.67 & 29.56 \\
3 (Panel $x+$ ) & 838.78 & 61.40 & 1.24 & 26.67 & 28.43 \\
4 (Panel $X-)$ & 842.85 & 61.70 & 1.25 & 26.67 & 28.57 \\
\hline
\end{tabular}

Table 7. Parameter a of the 1-diode/2-resistor circuit model extracted from the experimental results.

\begin{tabular}{llllll}
\hline & Panel $Y+$ & Panel $Y-$ & Panel $X+$ & Panel $X-$ & Panel $Z+$ \\
$a$ & 0.825 & 1.254 & 1.608 & 1.257 & 0.877 \\
\hline
\end{tabular}

The effects of atmospheric transmittance can be accounted for using the AM1.5G spectrum as usual. However, in order to obtain the most accurate results possible, the irradiance at each test realization is measured with a luxmeter placed in the same position and inclination as the solar panel under study.

The luxmeter provides the integral of the incident radiation spectrum reaching its sensor, by weighting the spectral irradiance with its photopic luminosity function $R_{\lambda}(\lambda)$, according to equation (4):

$$
E_{\nu}=\int_{\lambda} R_{\lambda}(\lambda) \hat{E}_{\lambda}(\lambda) \mathrm{d} \lambda
$$

where $E_{\nu}$ is the illuminance, in $\mathrm{Wm}^{-2}, \hat{E}_{\lambda}(\lambda)$ is the spectral irradiance reaching the luxmeter sensor and $R_{\lambda}$ is the photopic luminous efficacy of radiation, which has a maximum possible value of $683 \mathrm{~lm} \mathrm{~W}^{-1}$ for the case of monochromatic light at a wavelength of $555 \mathrm{~nm}$.

If the emission spectrum is assumed to remain constant, i.e. there is not radiation absorption for particular wavelengths between the luxmeter sensor and the light source (the sun), then the spectral irradiance reaching the panel could be expressed as 


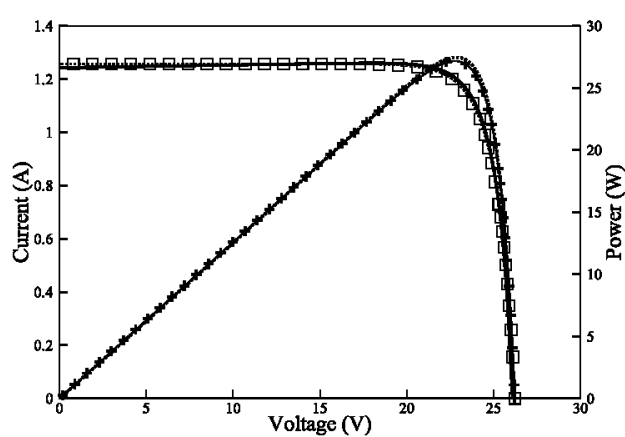

(a)

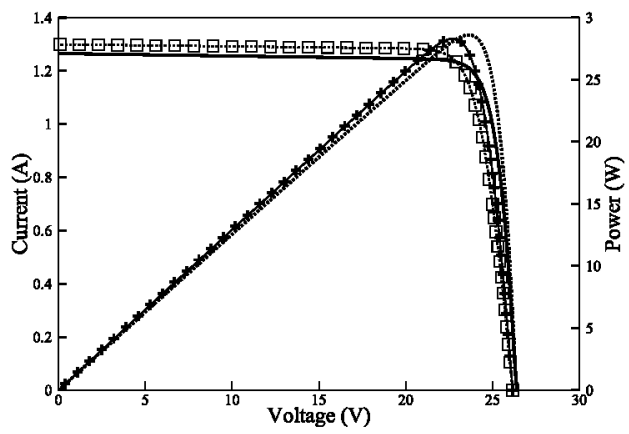

(c)

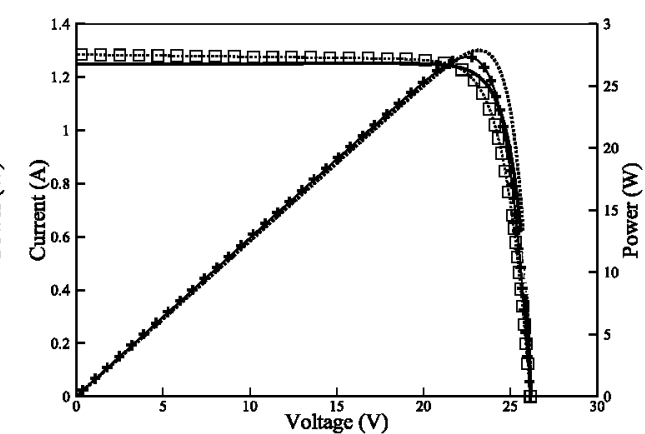

(b)

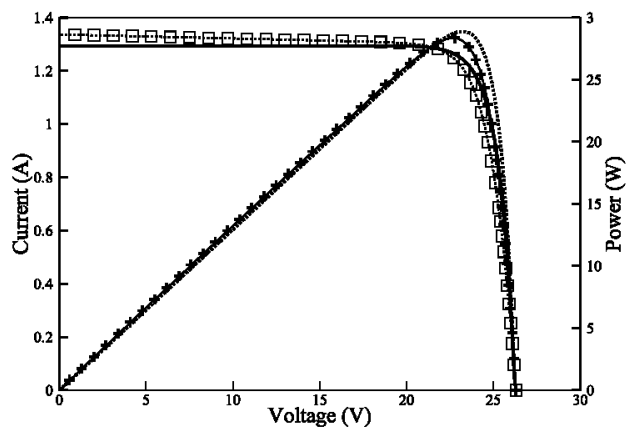

(d)

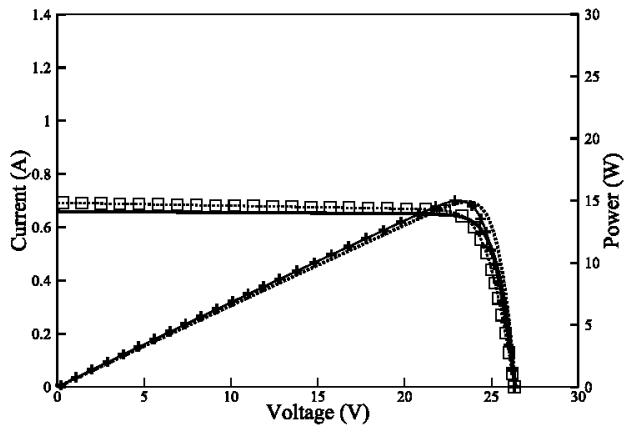

(e)

Figure 9. Comparison between the experimental $I-V$ and $P-V$ curves from the test and the resulting ones of the numerical model simulation from section 5.1, with spectral irradiance values from the testing conditions (see table 6). Figures from (a) to (e), three different solar panels of the UPMSAT-2 microsatellite. For all graphs: (- - - -) $I-V$ curves from the test measurements; ( - ) $I-V$ curves simulated with the 1-diode/2-resistor model; $(-+-) P-V$ curves from the test measurements; and (- - - $) P-V$ curves simulated with the 1-diode/2-resistor theoretical model. (a) Lateral solar panel $X+$. (b) Lateral solar panel $X-$. (c) Lateral solar panel $Y+$. (d) Lateral solar panel $Y-$. (e) Top solar panel $Z+$.

$$
\hat{E}_{\lambda}(\lambda)=C E_{\lambda, A M 1.5 G}(\lambda)
$$

where $C$ is a constant and $E_{\lambda, A M 1.5 G}(\lambda)$ is the normalized spectral irradiance of the sun in $\mathrm{AM} 1.5 \mathrm{G}$ conditions.

The value of $C$ can be calculated from equations (4) and (5). In the test conditions it varies between 0.95 and 1.01 .

To reference the spectral irradiance under test conditions to the extraterrestrial one, using the AM0 standard, the transmittance of the methacrylate plate protecting the solar panels, $\tau_{m}(\lambda)$, is used. The methacrylate plate is characterized by the measurements of its directional transmittance and hemispherical reflectance, using a spectrophotometer LAMBDA 950. Results of transmittance per wavelength are shown in figure 7.

The total spectral irradiance over the solar panels of equation (3), referred to the AMO spectrum is then

$$
E_{\lambda}(\lambda)=C \tau_{m}(\lambda) E_{\lambda, A M 1.5 G}(\lambda) .
$$

Values of spectral irradiance under test conditions are presented in figure 8(a) with grey lines, whereas the mean value is presented with a black line. In addition, the AMO spectrum is also included in figure 8 (a) with a black dotted line.

If the spectral irradiance of equation (6) is integrated in the whole wavelength range for the different test conditions, the total irradiance level reaching the solar cells referenced to the AM0 spectrum could be expressed as equation (7):

$$
\frac{\Phi_{i}}{\Phi_{\mathrm{AMO}}}=\frac{\int E_{\lambda, i}(\lambda) \mathrm{d} \lambda}{\int E_{\lambda, A M 0}(\lambda) \mathrm{d} \lambda}
$$

where the sub-index $i$ stands for the test ID, $\Phi_{i}$ is the total irradiance for test $i$, and $\Phi_{\mathrm{AM} 0}$ is the total irradiance for the AM0 


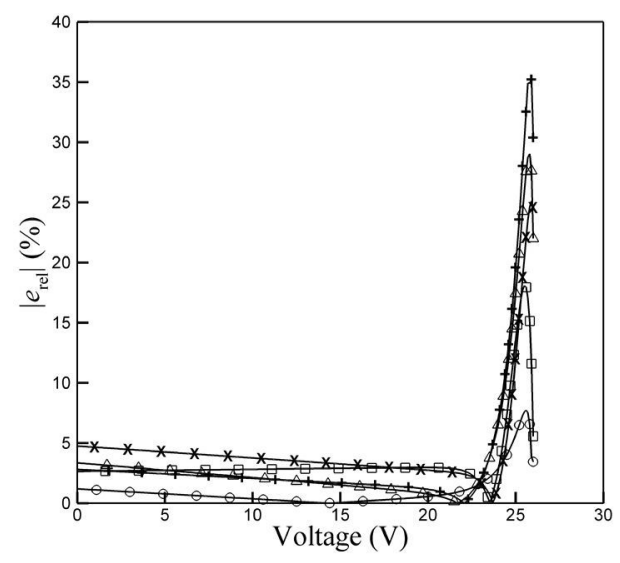

(a)

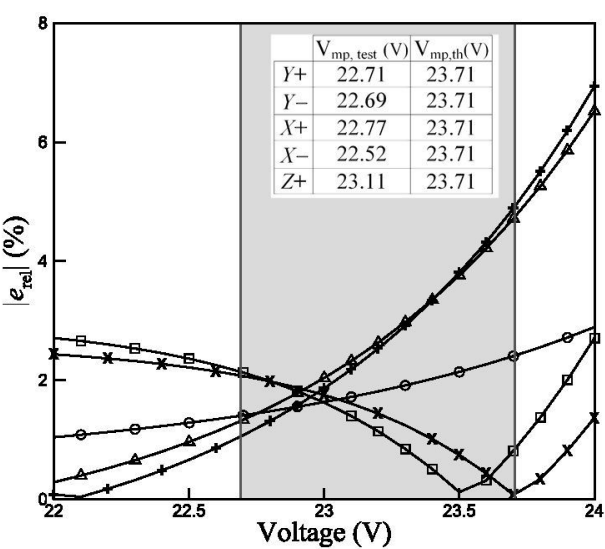

(b)

Figure 10. (a) Relative errors in percentage between the measured and simulated curves. $(-\square-)$ Panel $Y+;(-\triangle-)$ Panel $Y-$; (-o - ) Panel $X+$; (- + -) Panel $X-$; and $(-\times-$ ) Panel $Z+$. (b) A detail of graph (a) around the maximum power points.

spectrum that is $\Phi_{\mathrm{AM} 0}=1366.1 \mathrm{Wm}^{-2}$. Results of the irradiance levels for the different tests performed are presented in figure 8(b).

As can be extracted from figure $8(\mathrm{~b})$, the total irradiances during the test campaign are in the range of $806 \mathrm{Wm}^{-2}$ to $861 \mathrm{Wm}^{-2}$, accounting for $59-63 \%$ of the extraterrestial irradiance of the AM0 spectrum.

\section{Test measurement comparison with the theoretical model}

\subsection{Theoretical model description}

An ideal solar cell is usually described as a current source connected in parallel to an ideal diode [36], represented by the equation proposed by Shockley [31]. This model is commonly completed with resistors representing efficiency losses: a series resistance, $R_{s}$, accounting for the resistance of the materials which compose the module and causes a reduction on the power converted by the device [37-39]; and a shunt resistance, $R_{\text {sh }}$, connected in parallel with the diode, taking into account the alternative paths for the free carriers produced by the solar radiation [40-42].

The 1-diode/2-resistor model (or five parameter model) is the most popular equivalent circuit to represent solar panel behaviour and is governed by equation (8):

$$
I=I_{\mathrm{ph}}-I_{0}\left[\exp \left(\frac{V+I R_{s}}{n a V_{T}}\right)-1\right]-\frac{V+I R_{s}}{R_{\mathrm{sh}}}
$$

where $I_{\mathrm{ph}}$ is the photogenerated current by the cell, $I_{0}$ is the reverse saturation or leakage current and $V$ the applied voltage on cell terminals. As the solar panels are composed of 10 -series connected triple junction solar cells, the value of $n$ is set to 30 [29]. The parameter $V_{T}$ is the thermal voltage of the diode, governed by equation (9):

$$
V_{T}=k T / q
$$

where $q$ is the electron charge, $k$ the Boltzmann constant and $T$ the temperature.
Table 8. Root mean square error (RMSE) for each panel, calculated with the theoretical model (manufacturer's datasheet) and the experimental results from tests.

\begin{tabular}{ll}
\hline Panel & RMSE parameter \\
\hline$Y+$ & $1.60 \cdot 10^{-3}$ \\
$X+$ & $0.73 \cdot 10^{-3}$ \\
$Y-$ & $2.10 \cdot 10^{-3}$ \\
$X_{-}$ & $1.70 \cdot 10^{-3}$ \\
$Z+$ & $2.90 \cdot 10^{-3}$ \\
\hline
\end{tabular}

The non-dimensional constant of equation (8), $a$, is usually added to the $I-V$ curve, called the ideality factor, which takes into account the deviation of the diode from the Shockley diffusion theory. Usually, the value of this factor is assumed to be a constant between 1 and 1.5 [43].

To represent the $I-V$ curve the five parameters $\left(I_{\mathrm{ph}}, I_{0}, R_{S}\right.$, $R_{\mathrm{sh}}$ and $a$ ) must be identified [44]. Five boundary conditions are needed, usually extracted from the manufacturer's data, that are the value of the most representative point (short circuit, open circuit and maximum power points) and the temperature during characteristic points' measurements. In general, if an initial estimation of the ideality factor is used, then the other parameters could be calculated and $a$ may be adjusted afterwards [45].

For the UPMSAT-2 solar panels, if the manufacturer's datasheet (see table 3) is used, the values obtained for the model parameters are shown in table 5 .

With values of table 5 , the equation (8) defines the $I-V$ characteristic curves for the panels under study.

According to the traditional approach for the five parameter model, the photocurrent $I_{\mathrm{ph}}$ depends on the irradiance and shares a linear relationship with it [45]. To adjust the theoretical $I-V$ curves to the test insolation conditions, the value of $I_{\mathrm{ph}}$ is modified according to the measured incident irradiance level. In practice, the results of figure 8(b) are used to modify the value of $I_{\mathrm{ph}}$ to adjust the theoretical model to the test incident irradiance and, as a consequence, values of $I_{\mathrm{sc}}$ and $P_{\mathrm{mp}}$ are modified. 


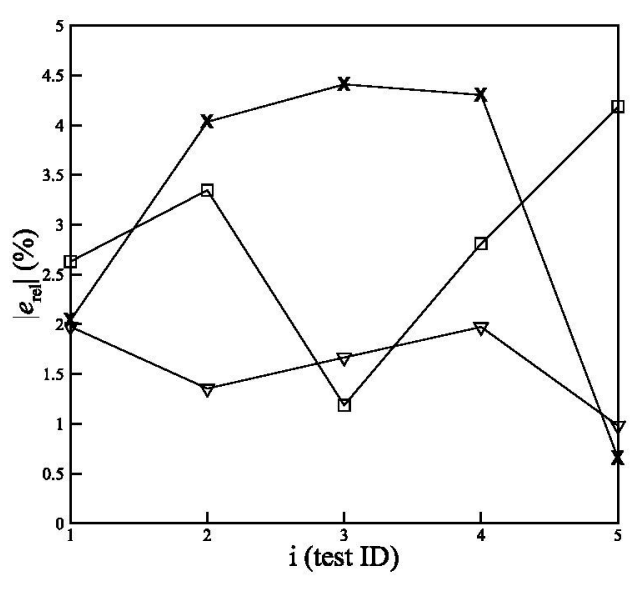

(a)

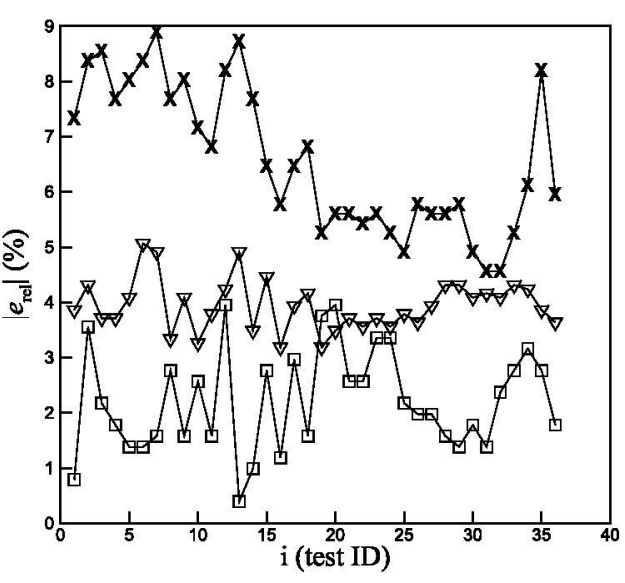

(b)

Figure 11. (a) Relative errors in the main $I-V$ parameters for (a) the testing conditions presented here and (b) the data provided by the solar modules manufacturer. For both graphs: (- $\square-) I s c ;(-\nabla-) V_{\mathrm{oc}}$; and $(-\times-) P_{\mathrm{mp}}$.

Values of $I_{\mathrm{sc}}, V_{\mathrm{oc}}$ and $P_{\mathrm{mp}}$ of the theoretical $I-V$ curves are presented in table 6 , along with the irradiance level $\left(\Phi_{i} / \Phi_{\mathrm{AM} 0}\right)$ used for their determination.

It should be noted that the value $V_{\mathrm{oc}}=26.67 \mathrm{~V}$ remains constant for every test. It is due to the nature of the theoretical model, which introduces no variation in the open circuit voltage with the incident irradiance. This fact is essentially true as extracted from previous works, where the $V_{\mathrm{oc}}$ parameter hardly varies with irradiance $[43,46]$. Indeed, the error introduced in the model, if variations of $V_{\mathrm{oc}}$ with irradiance levels are not taken into account, is lower than $0.5 \%$ for these works.

Once the theoretic $I-V$ curves are determined under test conditions, the parameter $a$ is adjusted using experimental results, as described in the following section.

\subsection{Theoretical model comparison with test results}

This section is focused on the comparison between the measured $I-V$ curves and the theoretical ones, calculated with the model described in section 5.1.

The theoretical $I-V$ curves calculated in the previous section are adjusted by using the parameter $a$ derived from the experimental results. To extract this parameter from the measured data, the experimental results were fitted to the model represented by equation (8) using a MATLAB ${ }^{\circledR}$ code, obtaining the results presented in table 7 .

The temperature of comparison is the manufacturer's reference one, $T=28^{\circ} \mathrm{C}$, so measured values are normalized to it as defined in section 4.2.

Figures 9(a) to (e) show the $I-V$ curves measured in the test campaign along with the $I-V$ curves obtained with the theoretical model. In addition, the $P-V$ curves are included for three solar panels of the UPMSAT-2.

The relative errors between the measured $I-V$ curves and those simulated with the 1-diode/2-resistor model are calculated and the obtained results are presented in figure 10(a). The relative error magnitude is calculated taking the measured current as the exact value, and the current calculated with the theoretical model (with the manufacturers' datasheet) as the approximation, i.e. $\left|e_{\text {rel }}\right|=\left|I_{\text {test }}-I_{\text {th }}\right| / I_{\text {test }}$.

As figure 10(a) shows, differences between the measured and simulated data using the manufacturer's datasheet remain within the usual values when a theoretical model is used $[45,47]$. A maximum in the relative error is observed around the open circuit point, reaching the largest value for panel $X-$, about $35 \%$. This result is due to the excessive relative error magnitude and takes into account the error around the open circuit point, where the solar panel current is nearly $0 \mathrm{~A}[45,48]$. However, in the operational point of the panel, i.e. in the vicinity of the maximum power point, the errors remain between 0.08 and less than $5 \%$. These values can be observed in figure 10(b), where the grey area represents the values of $V_{\mathrm{mp}}$ for both the manufacturer's and measured data.

To globally compare the results, the root mean square error (RMSE) is used as a criterion to quantify the difference between the model results and the experimental data [49]. RMSE is defined by equation (10).

$$
\mathrm{RMSE}=\sqrt{\frac{\boldsymbol{1}}{N} \sum_{i=1}^{N}\left(I_{\mathrm{th}, i}-I_{i}\right)^{2}}
$$

Values of RMSE are presented in table 8, for the different panels of the UPMSAT-2. These values are between RMSE $=0.73 \cdot 10^{-3}$ and RMSE $=2.9 \cdot 10^{-3}$. Based on the obtained RMSE, one can observe a good agreement between the experimental data and the model results.

To validate the testing conditions, the relative errors $\left|e_{\text {rel }}\right|$ between main parameters, i.e. short circuit current $I_{\mathrm{sc}}$, open circuit voltage $V_{\mathrm{oc}}$ and maximum power point $P_{\mathrm{mp}}$, of the solar panels are calculated for two different test cases: (i) The measured values within the testing conditions described here and; (ii) the experimental results provided by the solar modules manufacturer (Selex Galileo). The results for both cases are presented in figures 11(a) and (b). For the testing conditions presented here, the relative error remains between $0 \%$ and $5 \%$ while the measured data provided by the modules 
manufacturer are slightly larger. Indeed, for the relative errors of $I_{\mathrm{sc}}$ and $V_{\mathrm{oc}}$ the results are similar in both cases, whereas the maximum power point is better determined with the method proposed here.

These results, in addition to the RMSE values, also suggest that with a good knowledge of the characteristic points, the numerical model can accurately reproduce the experimental $I-V$ curve, so the assumptions that have been taken into account to normalize the experimental data have been selected adequately.

\section{Conclusions}

This paper proposes a method to conduct a solar panel performance test for small-size satellites' applications, by using an outdoor clean sky test environment. The key point to obtain good results is to properly monitor the testing conditions, i.e. the irradiance levels and solar cells' temperature, to be able to normalize measured data to the manufacturers' datasheet conditions that are usually referenced to the AM0 spectrum and a temperature of $T=28{ }^{\circ} \mathrm{C}$. The normalized $I-V$ curves obtained during the test campaign are compared with the $I-V$ curves calculated using a usual theoretical model (1-diode/2resistor). The $I-V$ curves simulated with the theoretical circuit model are based on the manufacturer's datasheet of the solar cells.

Relative errors between the measured and simulated $I-V$ curves and the root mean square errors are used to evaluate the quality of fit. As the RMSE parameter shows, the testing conditions used in this work can be applied to efficiently determine the main parameters of solar panels without the need for special and expensive test equipment. Indeed, the percentage deviation of the main parameters between the measured data and that extracted from the solar cells manufacturer are smaller for the test performed here than the data provided by the modules manufacturer.

Although it would be beyond the scope of this work to exhaustively analyse if the theoretical circuit model used is good enough to compute the $I-V$ curves using the manufacturer's data, the use of more complex theoretical models to acquire an accurate $I-V$ characteristic should improve the relative error obtained. Indeed, differences obtained between the test and experiment agreed with previous studies published in the literature $[45,48]$, confirming that the $I-V$ curves have been captured and post-processed adequately.

\section{Acknowledgments}

The present work was carried out in the framework of the UPMSAT-2 project at the IDR/UPM Institute (Madrid, Spain). The authors are indebted to the support of the staff of the IDR/ UPM Institute. The authors are also indebted to J Zamorano, J A de la Puente, A Alonso and J Garrido, from STRAST/ UPM, for their constant and kind support. The authors are truly grateful to J L Balenzategui, from the CIEMAT, for his useful help during the present research.

\section{ORCID iDs}

E Roibás-Millán ำ https://orcid.org/0000-0003-0180-6973

\section{References}

[1] Green M A, Emery K, Hishikawa Y, Warta W, Dunlop E D, Levi D H and Ho-Baillie A W Y 2017 Solar cell efficiency tables (version 49) Prog. Photovolt., Res. Appl. 25 3-13

[2] Swartwout M and Jayne C 2016 University-Class spacecraft by the numbers: success, failure, debris (but mostly success) 30th AIAA/USU Conf. on Small Satellites

[3] Rodiek J A and Brandhorst H W 2008 Solar array reliability in satellite operations 33rd IEEE Photovoltaic Specialists Conference, San Diego, CA, USA pp 1-4

[4] Shekoofa O 2011 Solar arrays testing for space applications Proc. 5th Int. Conf. Recent Advances in Space Technologies (RAST2011, Istanbul) pp 560-5

[5] Granek F and Danowicz T 2004 Advanced system for calibration and characterization of solar cells Opto-electron. Rev. 12 57-64

[6] Bucher K 1997 Calibration of solar cells for space applications Prog. Photovolt., Res. Appl. 5 91-107

[7] Berman D and Faiman D 1997 EVA browning and the timedependence of $I-V$ curve parameters on PV modules with and without mirror-enhancement in a desert environment Sol. Energy Mater. Sol. Cells 45 401-12

[8] Keogh W M and Blakers A W 2004 Accurate measurement, using natural sunlight, of silicon solar cells Prog. Photovolt., Res. Appl. 12 1-19

[9] ASTM G173-03 2012 Standard Tables for Reference Solar Spectral Irradiances: Direct Normal and Hemispherical on $37^{\circ}$ Tilted Surface (West Conshohocken, PA: ASTM International) (https://doi.org/10.1520/G0173-03R12)

[10] Dirnberger D, Krling U, Mllejans H, Salis E, Emery K, Hishikawa Y and Kiefer K 2014 Progress in photovoltaic module calibration: results of a worldwide intercomparison between four reference laboratories Meas. Sci. Technol. 25105005

[11] Carrillo J M, Martínez-Moreno F, Lorenzo C and Lorenzo E 2017 Uncertainties on the outdoor characterization of PV modules and the calibration of reference modules Sol. Energy 155 880-92

[12] Balenzategui J L, Rodríguez-Outón I and Chenlo F 2010 Calibration of crystalline silicon solar cells as reference devices for cell testers and sorters 5 th World Conference on Photovoltaic Energy Conversion, 6-10 September 2010, Valencia, Spain pp 2642-8

[13] Balenzategui J L, Cuenca J, Rodríguez-Outón I and Chenlo F 2012 Intercomparison and validation of solar cell I-V characteristic measurement procedures 27th European Photovoltaic Solar Energy Conference and Exhibition pp 1471-6

[14] Silva J P, Balenzategui J L and Nieto M B 2008 On the relationship between spectral reflectance and working temperature of PV modules $23 \mathrm{rd}$ European Photovoltaic Solar Energy Conference and Exhibition, Valencia, Spain pp 2861-4

[15] Silva J P, Nofuentes G G and Munoz J V 2010 Spectral reflectance patterns of photovoltaic modules and their thermal effects ASME. J. Sol. Energy Eng. 132041016

[16] Shaw G A D and Falconer J D 1971 SERT II-solar array power system $J$. Spacecr. Rockets 8 231-3

[17] Lumpp J K, Lumpp J E, Erb D M and Torabi N M 2010 The evaluation of solder and circuit board materials for small satellite solar cell arrays IEEE Aerospace Conference, Big Sky, $M T$ pp 1-6 
[18] Hoang B, Wong F K, Corey R L, Gardiner G, Funderburk V V, Gahart R L, Wright K H, Schneider T A and Vaughn J A 2012 Combined space environmental exposure tests of multi-junction GAAS/GE solar array coupons IEEE Trans. Plasma Sci. 40 324-33

[19] Scheiman D A and Snyder D B 2008 Low intensity low temperature (LILT) measurements of state-of-the-art triple junction solar cells for space missions $33 \mathrm{rd}$ IEEE Photovoltaic Specialists Conf. pp 1-6

[20] Scheiman D A, Piszczor M F, Snyder D B, McNatt J, Landis G A, Isabella L F and Putt N A 2011 Testing of solar cells for the solar probe plus mission 37th IEEE Photovoltaic Specialists Conf. pp 1620-5

[21] Ercol C J, Jenkins J, Dakermanji G, Santo A G and Mason L S 2000 Prototype solar panel development and testing for a Mercury orbiter spacecraft 35 th Intersociety Energy Conversion Engineering Conf. and Exhibit Collection of Technical Papers (Cat. No.00CH37022) vol 1, pp 449-59

[22] King D L and Kratochviland J A and Boyson W E 1997 Field experience with a new performance characterization procedure for photovoltaic arrays 2 nd World Conf. and Exhibition on Photovoltaic Solar Energy Conversion pp 1-8

[23] Malik A Q and Damit S J 2003 Outdoor testing of single crystal silicon solar cells Renew. Energy 28 1433-45

[24] Sanz-Andrés A and Meseguer J 1997 The programme of small satellites of the Universidad Politecnica de Madrid Selected Papers on Space Science Education, Remote Sensing, and Small Satellites. Seminars of the United Nations Programme on Space Applications vol 8 (New York: United Nations) p 168

[25] Sanz-Andrés A, López-Díez J, Espino J L, Marco Gómez V and Hernández L 1993 UPM/LB Sat: a small scientific educational satellite 7th Annual AIAA/Utah State University Conf. on Small Satellites vol 8, p 1

[26] Pindado S et al 2016 MUSE (Master in Space Systems), an advanced master's degree in space engineering Athens: ATINER'S Conf Paper Series vol 1, p 1953

[27] Markvart A T and Castaner L 2012 Practical Handbook of Photovoltaics: Fundamentals and Applications 2nd edn (Amsterdam: Elsevier)

[28] Tossa A K, Soro Y M, Azoumah Y and Yamegueu D 2014 A new approach to estimate the performance and energy productivity of photovoltaic modules in real operating conditions Sol. Energy $110543-60$

[29] Bana S and Saini R P 2016 A mathematical modeling framework to evaluate the performance of single diode and double diode based SPV systems Energy Rep. 2 171-87

[30] Fahrenbruch A L and Bube R H 1983 Fundamentals of Solar Cells: Photovoltaic Solar Energy Conversion (Oxford: Elsevier)

[31] Sze S M 1981 Physics of Semiconductor Devices (New York: Wiley)

[32] Whitfield K and Osterwald C R 2001 Procedure for determining the uncertainty of photovoltaic module outdoor electrical performance Prog. Photovolt., Res. Appl. $987-102$

[33] ASTM E490-00a 2014 Standard Solar Constant and Zero Air Mass Solar Spectral Irradiance Tables (West Conshohocken, PA: ASTM International)

[34] IEEE Standard 3071969 Standard definitions of terms for solar cells (Piscataway, NJ: IEEE)

[35] Iqbal M 1983 An Introduction to Solar Radiation (New York: Academic)

[36] Ciulla G, Lo Brano V, Di Dio V and Cipriani G 2014 A comparison of different one-diode models for the representation of $I-V$ characteristic of a PV cell Renew. Sustain. Energy Rev. 32 684-96

[37] Lineykin S, Averbukh M and Kuperman A 2014 An improved approach to extract the single-diode equivalent circuit parameters of a photovoltaic cell/panel Renew. Sustain. Energy Rev. $30282-9$

[38] Xiao W, Dunford W G and Capel A 2004 A novel modelling method for photovoltaic cells Proc. IEEE 35th Annual Power Electronics Specialist Conf. vol 3, p 1

[39] Chenni R, Makhlouf M, Kerbache T and Bouzid A 2007 A detailed modeling method for photovoltaic cells Energy 32 1724-30

[40] van Dyk E E and Meyer E L 2004 Analysis of the effect of parasitic resistances on the performance of photovoltaic modules Renew. Energy 29 333-44

[41] Carrero C, Rodríguez J, Ramírez D and Platero C 2010 Simple estimation of PV modules loss resistances for low error modelling Renew. Energy 35 1103-8

[42] Zhu X, Fu Z H, Long X and Li X 2011 Sensitivity analysis and more accurate solution of photovoltaic solar cell parameters Sol. Energy 85 393-403

[43] Villalva M G, Gazoli J R and Filho May E R 2009 Comprehensive approach to modeling and simulation of photovoltaic arrays IEEE Trans. Power Electron. 24 1198-208

[44] Ghani F, Rosengarten G, Duke M and Carson J K 2014 The numerical calculation of single-diode solar-cell modelling parameters Renew. Energy 72 105-12

[45] Cubas J, Pindado S and Victoria M 2014 On the analytical approach for modeling photovoltaic systems behavior J. Power Sources 247 467-74

[46] Cubas J, Pindado S and de Manuel C 2014 Explicit expressions for solar panel equivalent circuit parameters based on analytical formulation and the Lambert W-function Energies 7 4098-115

[47] Peng L, Sun Y, Meng Z, Wang Y and Xu Y 2013 A new method for determining the characteristics of solar cells J. Power Sources 227 131-6

[48] Pindado S, Cubas J and Sorribes-Palmer F 2015 On the analytical approach to present engineering problems: photovoltaic systems behavior, wind speed sensors performance, and high-speed train pressure wave effects in tunnels Math. Problems Eng. 2015 1-18

[49] Askarzadeh A and Rezazadeh A 2012 Parameter identification for solar cell models using harmony search-based algorithms Sol. Energy 86 3241-9 